\title{
Fauna de flebotomíneos (Diptera: Psychodidae) em um foco de leishmaniose tegumentar americana na área periurbana de Manaus, Estado do Amazonas
}

\author{
Sandfly fauna (Diptera: Psychodidae) in a focus of American cutaneous \\ leishmaniasis on the urban periphery of Manaus, State of Amazonas
}

\author{
Maria das Graças Vale Barbosa ${ }^{1,2,3}$, Nelson Ferreira Fé ${ }^{1}$, Alexandre Herculano Ribera Marcião ${ }^{4}$, \\ Ana Paula Thome Silva ${ }^{4}$, Wuelton Marcelo Monteiro ${ }^{1,2,5}$ \\ e Jorge Augusto de Oliveira Guerra ${ }^{1,2}$
}

\begin{abstract}
RESUMO
No período de agosto de 2001 a julho de 2002, usando armadilhas CDC e Disney, realizaram-se coletas de flebotomíneos, na base de árvores no peridomicílio e nas matas da Comunidade São João, área periurbana de Manaus, Amazonas. Foram capturados 4.104 espécimes, pertencentes a quatro subtribos, 13 gêneros e 49 espécies da subfamília Phlebotominae. Predominou a subtribo Psychodopygina com 3.403 (83\%) espécimes, destacandose Nyssomyia umbratilis, Nyssomyia anduzei, Trichophoromyia eurypyga, Bichromomyia olmeca nociva e Bichromomyia flaviscutellata. 0 registro de Nyssomyia umbratilis e Nyssomyia anduzei, incriminadas como vetoras de Leishmania (Viannia) guyanensis, e de Bichromomyia flaviscutellata e Bichromomyia olmeca nociva, de Leishmania (Leishmania) amazonensis, indicam risco de infecção para os moradores da área. A grande maioria (98,5\%) dos flebotomíneos foi capturada na área de mata. Nyssomyia anduzei e Bichromomyia olmeca nociva foram coletadas no peridomicílio. A riqueza de espécies vetoras de Leishmania nessa área revela a necessidade de uma vigilância entomológica constante.
\end{abstract}

Palavras-chaves: Phlebotominae. Leishmaniose tegumentar americana. Diversidade de espécies.

\begin{abstract}
From August 2001 to July 2002, sand flies were collected from the bases of trees and, using CDC and Disney traps, from areas surrounding homes and forested areas in the São João community, on the urban periphery of Manaus, State of Amazonas. 4,104 specimens belonging to four subtribes, 13 genera and 49 species of the Phlebotominae subfamily were collected. The subtribe Psychodopygina predominated, with 3,403 (83\%) specimens, especially of Nyssomyia umbratilis, Nyssomyia anduzei, Trichophoromyia eurypyga, Bichromomyia olmeca nociva and Bichromomyia flaviscutellata. The occurrences of Nyssomyia umbratilis and Nyssomyia anduzei, which have been incriminated as vectors for Leishmania (Viannia) guyanensis, and of Bichromomyia flaviscutellata and Bichromomyia olmeca nociva, for Leishmania (Leishmania) amazonensis, indicate that there is a risk of infection for people living in this area. Most (98.5\%) of the sand flies were caught in the forested area. Nyssomyia anduzei and Bichromomyia olmeca nociva were collected from areas surrounding homes. The richness of vector species for Leishmania in this area shows the need for constant entomological surveillance.
\end{abstract}

Key-words: Phlebotominae. American cutaneous leishmaniasis. Species diversity.

A incidência da leishmaniose tegumentar americana (LTA) tem aumentado na América Latina, principalmente no Brasil, onde o número de casos chegou a 610.256, de 1980 a 2005. Nesse período, realizaram-se 226.252 notificações na Região Norte, representando $37,1 \%$ dos casos do Brasil, dos quais $50.740(22,4 \%)$ ocorreram no Estado do Amazonas ${ }^{28}$. Neste estado, a incidência da LTA vem aumentando significativamente, acompanhando principalmente a instalação de novos núcleos residenciais em áreas onde previamente existia densa floresta tropical $^{22}$.

No município de Manaus, onde se registra mais da metade dos casos de LTA do Estado do Amazonas ${ }^{102}$, há nítido predomínio da doença causada pela Leishmania (Viannia) guyanensis, cujo principal transmissor é Nyssomyia umbratilis, sendo

1. Gerência de Entomologia e Leishmaniose, Fundação de Medicina Tropical do Amazonas, Manaus, AM. 2. Escola Superior de Ciências da Saúde, Universidade do Estado do Amazonas, Manaus, AM. 3. Centro Universitário Nilton Lins, Manaus, AM. 4. Faculdade de Medicina, Universidade Federal do Amazonas, Manaus, AM. 5. Secretaria Estadual de Saúde do Amazonas, Manaus, AM.

Endereço para correspondência: Dra. Maria das Graças Vale Barbosa. Gerência de Entomologia/FMTAM. Av. Pedro Teixeira 25, Dom Pedro, 69040-000 Manaus, AM.

Fax: 5592 3656-8269

e-mail: gbarbosa@fmt.am.gov.br

Recebido em: 02/04/2008

Aceito em 08/07/2008 
Nyssomyia anduzei um vetor secundário ${ }^{319202430}$. A Leishmania (Leishmania) amazonensis é outro agente de LTA na região, cujos transmissores são Bichromomyia flaviscutellata e Bichromomyia olmeca nociva ${ }^{330}$. Neste município, principalmente na periferia, o grau de exposição dos indivíduos acometidos está relacionado diretamente a processos de ocupação desordenada ou às invasões ${ }^{1022}$, Assim sendo, geralmente a LTA ocorre em lugares de assentamentos populacionais recentes $^{2} 1013$. Possivelmente a transmissão também ocorra no intra e peridomicílio na região periurbana e rural de Manaus, pela proximidade das residências com as áreas de floresta, que pode ser considerado como um padrão periflorestal ${ }^{102123}$.

0 processo de modificação ambiental, decorrente da ação antrópica alterou os habitats dos flebotomíneos, resultando num aumento do risco de transmissão da LTA, devido à adaptação de algumas espécies ao ambiente modificado. Na região Amazônica, foram realizados vários estudos enfatizando os aspectos biológicos, taxonômicos e ecológicos dos flebotomíneos, observando-se que as alterações ocorridas em áreas de floresta influenciam na composiçã̃o e comportamento da fauna flebotomínica ${ }^{4511} 1233$.

Grande parte dos casos de LTA registrados em Manaus provém de áreas rurais adjacentes à rodovia $\mathrm{AM}-010$, que liga 0 município de Manaus aos de Rio Preto da Eva e Itacoatiara e à BR-174, que liga Manaus ao município de Presidente Figueiredo e ao Estado de Roraima ${ }^{2123}$. No período em que esse trabalho foi realizado, foram diagnosticados 17 casos de LTA na Comunidade São João ${ }^{23}$. Este fato demonstra a necessidade de se estudar a diversidade da fauna e a frequiência de flebotomíneos no ambiente domiciliar e peridomiciliar e nas matas remanescentes, nestas localidades endêmicas, tendo em vista a busca de meios para diminuir o contato entre homem e vetor.

\section{MATERIAL E MÉTODOS}

Área de estudo. Aárea estudada compreendeu a Comunidade de São João, no km 4 da BR-174, que liga Manaus a Boa Vista (RR), no município de Manaus ( $\left.03^{\circ} 08^{\prime} 07^{\prime \prime} \mathrm{s}, 60^{\circ} 01^{\prime} 34^{\prime \prime} 0\right)$. Nessa área, viviam aproximadamente 450 famílias, distribuídas em uma área aproximada de $500 \mathrm{~m}$ de largura por $1.200 \mathrm{~m}$ de comprimento. Limita-se a oeste com a Rodovia BR-174; à leste e ao sul, com áreas de floresta primária, semifragmentada, devido às atividades de extração madeireira; e ao norte, com uma área sem cobertura vegetal, onde são desenvolvidas atividades de avicultura e piscicultura. As matas vizinhas da comunidade são freqüentadas por muitos moradores para o desenvolvimento de atividades de extrativismo. Seu clima é tropical chuvoso e úmido, com temperatura média de $26^{\circ} \mathrm{C}$.

Coleta e identificação dos flebotomíneos. As coletas de flebotomíneos foram realizadas durante doze meses consecutivos, de agosto 2001 a julho 2002, no domicílio, peridomicílio e nas matas. As residências foram construídas muito próximas à área de mata, em alguns casos, até cerca de $20 \mathrm{~m}$, em outros, entre 100 e $500 \mathrm{~m}$. Foram programados cinco dias consecutivos de coleta em cada mês. Empregaram-se coletas ativas, usando rede entomológica e tubos de vidro e coletas passivas, utilizando cinco armadilhas de luz do tipo $\mathrm{CDC}^{38}$ (modificada), colocadas a um metro acima do solo, e armadilhas Disney ${ }^{39}$. As coletas ativas no intradomicílio e peridomicílio foram realizadas em dois dos cinco dias do período mensal de coletas, no horário de 18:00 às 21:00h. As coletas na base de árvores foram realizadas do segundo ao quarto dia, durante três horas diárias pela manhã, de 8:00 às 11:00h, em diversos pontos dentro da mata delimitada para execução do estudo.

As coletas passivas na mata, utilizando armadilhas $\mathrm{CDC}$, foram realizadas durante cinco dias consecutivos, iniciando às 17:00h e encerrando às $08: 00 \mathrm{~h}$ do dia seguinte; no peridomicílio, as armadilhas foram colocadas ao lado das casas para as capturas noturnas. As armadilhas Disney foram colocadas a partir do primeiro dia, trocando-se as bandejas untadas com óleo mineral diariamente às 08:00h e retirando-as no quinto dia.

Todo material foi acondicionado em caixa de isopor e levado para triagem e identificação pelo segundo autor, no Laboratório de Entomologia da Fundação de Medicina Tropical do Amazonas, onde os espécimes estão depositados. A nomenclatura das espécies segue Galati ${ }^{17}$.

As informações sobre a precipitação pluviométrica mensal no município de Manaus para o período do estudo foram fornecidas pelo $1^{\circ}$ Distrito de Meteorologia de Manaus, órgão do Instituto Nacional de Meteorologia.

Os dados analisados são apresentados de forma descritiva, incluindo-se análises da correlação entre a abundância de espécies vetoras de leishmaniose e a média mensal da precipitação pluviométrica, através do Coeficiente de Pearson.

\section{RESULTADOS}

Foram capturados 4.104 espécimes, pertencentes a quatro subtribos, 13 gêneros e 49 espécies da subfamília Phlebotominae. 0 predomínio entre as subtribos foi nitidamente de Psychodopygina com 3.403 (83\%) espécimes, destacando-se as espécies Nyssomyia umbratilis, com 1.325 (32,3\%) espécimes, Nyssomyia anduzei, com 820 (20\%), Trichophoromyia eurypyga, com 492 (12\%), Bichromomyia olmeca nociva, com 207 (5\%) e Bichromomyia flaviscutellata, com 185 (4,5\%). Foram coletados 613 (15\%) flebotomíneos da subtribo Lutzomyiina, a segunda com maior número de espécimes. Dentre eles, Trichopygomyia trichopyga foi a espécie mais freqüente 191 (4,7\%), seguida por Sciopemyia sordellii 81 (2\%), Pressatia triacantha 72 (1,8\%) e Evandromyia monstruosa 70 $(1,7 \%)$. Da subtribo Sergentomyiina, foram coletados 84 (2\%) flebotomíneos, com destaque para Micropygomyia rorotaensis $(1,9 \%)$. De Brumptomyiina, foram coletados quatro espécimes de Brumptomyia pintoi (Tabela 1).

Verificou-se que do total de flebotomíneos, 4.044 (98,5\%) espécimes foram capturados no interior da mata e $60(1,5 \%)$ no peridomicílio. Do total de espécies, 38 (77,6\%) foram coletadas apenas no interior das matas e $11(22,4 \%)$ foram coletadas tanto no interior das matas quanto no peridomicílio. Nenhum flebotomíneo foi capturado no intradomicílio. 
Tabela 1 - Espécies de flebotomíneos coletadas na Comunidade de São João, Manaus, Amazonas, de agosto de 2001 a julbo de 2002.

\begin{tabular}{|c|c|c|c|c|c|c|c|}
\hline \multirow[b]{2}{*}{ Espécies } & \multicolumn{2}{|c|}{ Peridomicílio } & \multicolumn{3}{|c|}{ Mata } & \multicolumn{2}{|c|}{ Total } \\
\hline & $\mathrm{CDC}$ & Manual & Base da árvore & $\mathrm{CDC}$ & Disney & $\mathrm{n}^{0}$ & $\%$ \\
\hline Bichromomyia flaviscutellata (Mangabeira) & 0 & 0 & 1 & 144 & 40 & 185 & 4,5 \\
\hline Bichromomyia olmeca nociva (Young e Arias) & 1 & 0 & 0 & 201 & 5 & 207 & 5,0 \\
\hline Brumptomyia pintoi (Costa Lima) & 0 & 0 & 0 & 4 & 0 & 4 & 0,1 \\
\hline Evandromyia andersoni ( Le Point e Desjeux) & 0 & 0 & 0 & 2 & 0 & 2 & 0,0 \\
\hline Evandromyia georgii (Freitas e Barret) & 0 & 2 & 0 & 16 & 0 & 18 & 0,4 \\
\hline Evandromyia infraspinosa (Mangabeira) & 0 & 0 & 0 & 22 & 1 & 23 & 0,6 \\
\hline Evandromyia inpai (Young e Arias) & 0 & 0 & 0 & 6 & 1 & 7 & 0,2 \\
\hline Evandromyia monstruosa (Floch e Abonnenc) & 0 & 0 & 0 & 70 & 0 & 70 & 1,7 \\
\hline Evandromyia sericea (Floch e Abonnenc) & 0 & 0 & 0 & 4 & 0 & 4 & 0,1 \\
\hline Evandromyia walkeri (Newstead) & 0 & 0 & 0 & 1 & 0 & 1 & 0,0 \\
\hline Evandromyia williamsi (Damasceno, Causey e Arouck) & 0 & 0 & 0 & 4 & 0 & 4 & 0,1 \\
\hline Lutzomyia evangelistai (Martins e Fraiha) & 0 & 0 & 0 & 1 & 0 & 1 & 0,0 \\
\hline Lutzomyia gomezi (Nitzulescu) & 0 & 0 & 11 & 44 & 0 & 55 & 1,3 \\
\hline Lutzomyia spathotrichia (Martins, Falcão e Silva) & 0 & 0 & 0 & 12 & 0 & 12 & 0,3 \\
\hline Micropygomyia pilosa (Damasceno e Causey) & 0 & 1 & 0 & 3 & 0 & 4 & 0,1 \\
\hline Micropygomyia rorotaensis (Floch e Abonnenc) & 0 & 0 & 3 & 76 & 0 & 79 & 1,9 \\
\hline Micropygomyia trinidadensis (Newstead) & 0 & 0 & 0 & 1 & 0 & 1 & 0,0 \\
\hline Nyssomyia anduzei (Rozeboom) & 1 & 0 & 19 & 799 & 1 & 820 & 20,0 \\
\hline Nyssomyia antunesi (Coutinho) & 0 & 0 & 1 & 16 & 1 & 18 & 0,4 \\
\hline Nyssomyia umbratilis (Ward e Fraiha) & 20 & 4 & 459 & 842 & 0 & 1.325 & 32,3 \\
\hline Pressatia triacantha (Mangabeira) & 0 & 1 & 0 & 71 & 0 & 72 & 1,8 \\
\hline Pressatia trispinosa (Mangabeira) & 0 & 0 & 0 & 47 & 0 & 47 & 1,1 \\
\hline Psathyromyia abonnenci (Floch e Chassignet) & 0 & 0 & 0 & 3 & 0 & 3 & 0,1 \\
\hline Psathyromyia barrettoi barrettoi (Mangabeira) & 0 & 0 & 0 & 1 & 0 & 1 & 0,0 \\
\hline Psathyromyia cuzquena (Martins, Llanos e Silva) & 0 & 0 & 0 & 2 & 0 & 2 & 0,0 \\
\hline Psathyromyia dendrophyla (Mangabeira) & 0 & 1 & 4 & 4 & 0 & 9 & 0,2 \\
\hline Psathyromyia lutziana (Costa Lima) & 0 & 0 & 0 & 2 & 0 & 2 & 0,0 \\
\hline Psathyromyia scaffi (Damasceno e Arouck) & 0 & 0 & 3 & 1 & 0 & 4 & 0,1 \\
\hline Psathyromyia shannoni (Dyar) & 0 & 0 & 4 & 4 & 0 & 8 & 0,2 \\
\hline Psathyromyia. aragaoi (Costa Lima) & 0 & 0 & 0 & 27 & 0 & 27 & 0,7 \\
\hline Psychodopygus amazonensis (Root) & 0 & 0 & 0 & 3 & 0 & 3 & 0,1 \\
\hline Psychodopygus chagasi (Costa Lima) & 0 & 0 & 0 & 3 & 0 & 3 & 0,1 \\
\hline Psychodopygus claustrei (Abonnenc, Léger e Fauran) & 0 & 0 & 0 & 9 & 0 & 9 & 0,2 \\
\hline Psychodopygus davisi (Root) & 0 & 0 & 0 & 150 & 4 & 154 & 3,8 \\
\hline Psychodopygus geniculatus (Mangabeira) & 0 & 0 & 1 & 11 & 0 & 12 & 0,3 \\
\hline Psychodopygus hirsutus hirsutus (Mangabeira) & 2 & 0 & 0 & 36 & 0 & 38 & 0,9 \\
\hline Psychodopygus paraensis (Costa Lima) & 0 & 0 & 0 & 1 & 0 & 1 & 0,0 \\
\hline Psychodopygus squamiventris squamiventris (Lutz e Neiva) & 0 & 0 & 0 & 5 & 0 & 5 & 0,1 \\
\hline Psychodopygus. ayrozai (Barreto e Coutinho) & 0 & 0 & 0 & 1 & 0 & 1 & 0,0 \\
\hline Psychodopygus. corossoniensis (Le Point e Pajot) & 0 & 0 & 0 & 2 & 0 & 2 & 0,0 \\
\hline Sciopemyia nematoducta (Young e Arias) & 0 & 0 & 0 & 24 & 0 & 24 & 0,6 \\
\hline Sciopemyia sordellii (Shannon e Del Ponte) & 1 & 6 & 0 & 74 & 0 & 81 & 2,0 \\
\hline Trichophoromyia eurypyga (Martins, Falcão e Silva) & 18 & 1 & 0 & 472 & 1 & 492 & 12,0 \\
\hline Trichophoromyia ruii (Arias e Young) & 0 & 0 & 0 & 8 & 0 & 8 & 0,2 \\
\hline Trichophoromyia ubiquitalis (Mangabeira) & 0 & 0 & 0 & 31 & 0 & 31 & 0,8 \\
\hline Trichopygomyia longispina (Mangabeira) & 0 & 0 & 0 & 1 & 0 & 1 & 0,0 \\
\hline Trichopygomyia trichopyga (Floch e Abonnenc) & 1 & 0 & 0 & 190 & 0 & 191 & 4,7 \\
\hline Viannamyia furcata (Mangabeira) & 0 & 0 & 0 & 26 & 0 & 26 & 0,6 \\
\hline Viannamyia tuberculata (Mangabeira) & 0 & 0 & 0 & 7 & 0 & 7 & 0,2 \\
\hline Total & 44 & 16 & 506 & 3.484 & 54 & 4.104 & 100,0 \\
\hline
\end{tabular}

CDC: armadilha de luz

No peridomicílio, a armadilha CDC coletou 44 (1,1\%) espécimes, enquanto $16(0,4 . \%)$ foram capturados nas coletas ativas, com destaque para as espécies Nyssomyia umbratilis, Trychophoromyia eurypyga, Bichromomyia olmeca nociva, Nyssomyia anduzei (Tabela 1).
No interior da mata, maior número de flebotomíneos foi capturado nas armadilhas CDC (3.484 espécimes), seguidas pelas coletas ativas nas bases das árvores (506) e pelas armadilhas Disney (54). Nas coletas passivas com armadilhas CDC foram registrados 3.484 flebotomíneos, destacando-se as 
espécies Nyssomyia umbratilis (842), Nyssomyia anduzei (798), Bichromomyia olmeca nociva (201) e Bichromomyia flaviscutellata (144). Nas coletas ativas na base das árvores destacam-se as espécies Nyssomyia umbratilis, (459 espécimes) e Nyssomyia anduzei (19). Nas coletas com armadilhas Disney, as espécies mais frequientes foram Bichromomyia flaviscutellata (39) e Bichromomyia olmeca nociva (5).

A distribuição temporal das quatro espécies incriminadas como vetoras mais freqüentes demonstrou que estas ocorreram durante todos os meses do ano (Figura 1). Nyssomyia umbratilis e Nyssomyia anduzei ocorreram durante todo o ano com maior número entre novembro e maio, quando ocorreu maior precipitação pluviométrica; Bichromomyia flaviscutellata e Bichromomyia olmeca nociva foram mais abundantes nos meses de abril a julho. Observou-se uma correlação positiva entre a precipitação pluviométrica mensal e variação temporal da abundância de Nyssomyia umbratilis $(\mathrm{r}=0,692$, $\mathrm{p}$-valor $=0,013)$ e Nyssomyia anduzei $(\mathrm{r}=0,664$, $\mathrm{p}$-valor=0,018), o que não foi verificado para Bichromomyia olmeca nociva $(\mathrm{r}=-0,439$, $\mathrm{p}$-valor $=0,154)$ e para Bichromomyia flaviscutellata $(\mathrm{r}=0,162$, p-valor $=0,616)($ Figura 2).
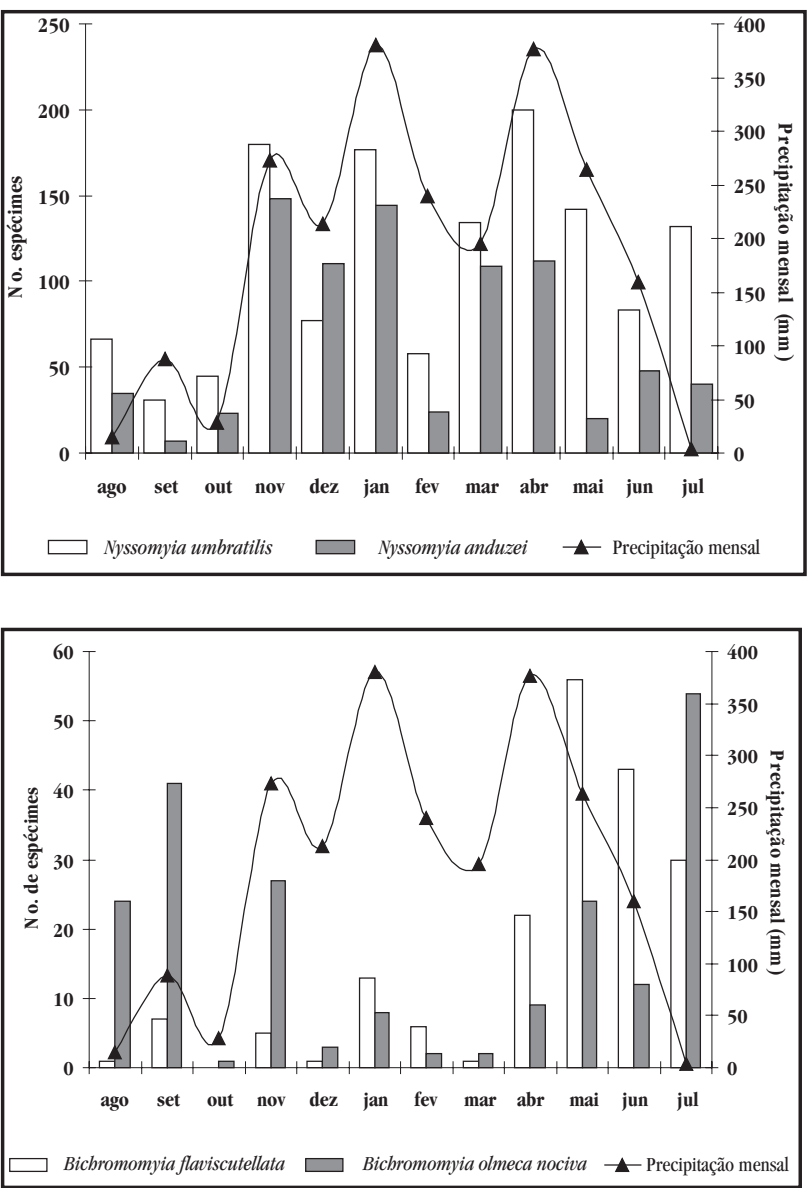

Figura 1 - Precipitação pluviométrica mensal e variação temporal da abundância das principais espécies de flebotomíneos envolvidas na transmissão de leishmaniose tegumentar americana na Comunidade de São João, Manaus, Amazonas, de agosto de 2001 a julbo de 2002.
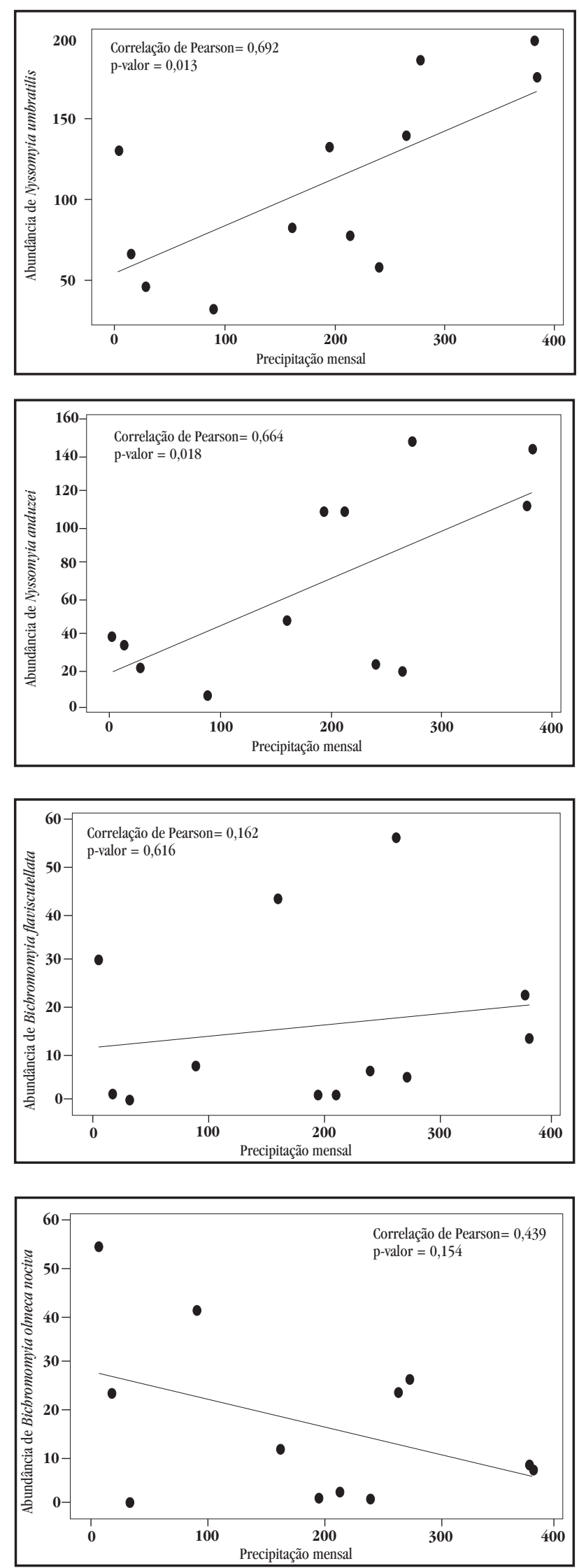

Figura 2 - Correlação entre a precipitação pluviométrica mensal e variação temporal da abundância das principais espécies de flebotomíneos envolvidas na transmissão de leishmaniose tegumentar americana na Comunidade São João, Manaus, Amazonas, de agosto de 2001 a julbo de 2002. 


\section{DISCUSSÃo}

Os dados obtidos evidenciaram uma fauna flebotomínica diversificada e abundante. As espécies assinaladas na Comunidade São João já o foram anteriormente no Estado do Amazonas 456812141536 , exceto Evandromyia andersoni. Esta espécie não antropofílica foi descrita na Bolívia ${ }^{27}$ e, no Brasil, existe o registro da sua ocorrência no Estado do Mato Grosso ${ }^{29}$. Destaca-se a importância deste relato sobre seu primeiro registro na Região Norte do Brasil.

A abundância de espécies dos gêneros Nyssomyia, Bichromomyia, Trichophoromyia e Psychodopygus, vetores de Leishmania, demonstraram risco para infecção humana nas matas da localidade. São gêneros predominantes na Região Amazônica, com diversidade de espécies maior do que em outras regiões do Brasil, incluindo espécies incriminadas como vetoras de LTA, tanto no ciclo enzoótico, quanto no ciclo zoonótico ${ }^{18}{ }^{19}$. A menor abundância de espécimes das espécies de Psychodopygus (5,6\%), em comparação com Nissomyia (52,7\%), estão de acordo com os resultados de coletas realizadas em Manacapuru, município limítrofe de Manaus ${ }^{36}$. Porém difere dos resultados encontrados em áreas de floresta primária amazônica, onde predominou Psychodopygus ${ }^{453435}$.

A espécie predominante tanto no ambiente florestal quanto no peridomiciliar, foi Nyssomyia umbratilis, principal vetora de Leishmania guyanensis ${ }^{624}$, responsável pela maioria casos de LTA na região Amazônica. Pode ser encontrada em grande quantidade no estágio de repouso sobre o tronco das árvores ${ }^{9}$ e apesar de sua principal atividade hematofágica ser crepuscular e noturna, pode atacar o homem nas primeiras horas do amanhecer. Em Manaus, predomina em regiões de florestas de terra firme ${ }^{34516}$. A espécie Nyssomyia anduzei, incriminada como vetor secundário da leishmaniose cutânea causada pela Leismania guyanensis na Região Amazônica ${ }^{326}$, foi a segunda mais abundante.

No gênero Trichophoromyia, destacou-se Trichophoromyia eurypyga, incluindo 19 espécimes no ambiente peridomiciliar. Apesar de nunca ter sido registrada sua infecção natural por parasitos do gênero Leishmania, este flebotomíneo tem sido coletado no Estado do Amazonas ${ }^{36}$, requerendo estudos que venham esclarecer seu papel na epidemiologia da leishmaniose neste Estado. A espécie Trichophoromyia ubiquitalis, apesar de ter sido coletada em menor quantidade e apenas no ambiente florestal, já foi encontrada naturalmente infectada por Leishmania lainsoni ${ }^{2537}$.

As espécies Bichromomyia olmeca nociva e Bichromomyia flaviscutellata foram mais frequientes no ambiente florestal. Um exemplar de Bichromomyia olmeca nociva foi coletado no peridomicílio. Bichromomyia flaviscutellata é incriminada como vetora de Leishmania (Leishmania) amazonensis, causadora de formas cutâneas e difusas da leishmaniose. Foi a espécie mais coletada nas armadilhas Disney, corroborando a eficiência deste método para coleta dessa espécie. Bichromomyia olmeca nociva já foi encontrada naturalmente infectada com Leishmania amazonensis em coletas realizadas na área periurbana de Manaus?.
Para o gênero Psychodopygus destaca-se Psychodopygus davisi, cuja distribuição geográfica abrange toda a Amazônia, é uma espécie envolvida na transmissão de Leishmania naiffi, da qual também já foi isolada Leishmania (Viannia) braziliensis $^{19}$. Psychodopygus ayrozai, Psychodopygus paraensis e Psychodopygus squamiventris squamiventris também foram referidos como transmissores de Leishmania naiffi ${ }^{31}$. Neste gênero, apenas Psychodopygus hirsutus hirsutus foi observado no ambiente peridomiciliar, esta espécie já foi encontrada infectada por Leishmania (Viannia) braziliensis ${ }^{32}$.

No peridomicílio, foram assinaladas 11 espécies de flebotomíneos. Feitosa e cols ${ }^{5}$ registraram 16 e Paes $^{30} 13$ espécies no peridomicílio na região de Manaus, em áreas de transmissão de LTA $^{1530}$. 0 encontro de espécimes de Psychodopygus hirsutus birsutus, Nyssomyia anduzei, Psathyromyia dendrophyla no peridomicílio pode ser porque estas espécies têm como habitats áreas marginais e anexos de animais domésticos ${ }^{1}$. Destaca-se o encontro de Evandromyia georgii, Bichromomyia olmeca nociva, Micropygomyia pilosa, Pressatia triacantha, e Trichopygomyia trichopyga. Considerando que essas espécies têm como principais habitats tocas de animais silvestres, folhas, troncos e raízes tabulares, seu encontro no peridomicílio juntamente com Nyssomyia umbratilis, pode ser devido à proximidade das residências com as áreas de mata, merecendo maiores estudos para verificar seu real potencial de domiciliação na área. Embora com número reduzido de indivíduos, algumas espécies de flebotomíneos podem estar se adaptando aos ambientes antrópicos, pois foram detectadas em todos os ambientes de coleta deste estudo.

A importância do padrão periflorestal de transmissão na Cidade de Manaus já foi ressaltada anteriormente ${ }^{210}$. Guerra e cols ${ }^{23}$ sugerem que na localidade do estudo ocorrem dois diferentes padrões epidemiológicos de transmissão de LTA. 0 primeiro, de transmissão silvestre, devido à penetração dos moradores da comunidade na mata para a realização de atividades extrativistas. Um segundo padrão, periflorestal, com um ciclo de transmissão no peridomicílio e nas áreas marginais em áreas de ocupação situadas dentro do raio de vôo dos vetores silvestres ${ }^{2}{ }^{10}$. Entretanto, a maioria dos casos de infecção por Leishmania guyanensis é contraída dentro da floresta, durante o dia, quando espécimes de Nyssomyia umbratilis são perturbados nos seus refúgios nas bases dos troncos das árvores ${ }^{36}$, principalmente em atividades de desmatamento ${ }^{24}$.

As observações sobre sazonalidade sugerem que as espécies coletadas têm grande plasticidade, com populações ora predominando em períodos mais secos, ora em períodos mais chuvosos. Registrou-se que Nyssomyia umbratilis e Nyssomyia anduzei foram muito freqüentes durante o ano todo, especialmente nos meses com maior precipitação pluviométrica, permitindo-se inferir que a população está mais sujeita à infecção pela Leishmania guyanensis nestes meses. A distribuição sazonal dos casos humanos na região de Manaus mostra maior número de casos no período chuvoso, onde se observa incremento dessas populações de vetores ${ }^{2230}$. Entretanto, as espécies Bichromomyia flaviscutellata e Bichromomyia olmeca nociva ocorreram 
em períodos relativamente mais curtos, e sua distribuição não mostrou relação com a pluviosidade. 0 registro de maior frequiência de Bichromomyia flaviscutellata, entre maio e julho, confirmam trabalhos realizados em áreas situadas na periferia da cidade de Manaus, dentre elas a Reserva Florestal Adolpho Ducke e áreas do bairro da Cidade Nova? .

A riqueza de espécies relacionadas à transmissão de LTA nessa área, revela a necessidade de uma vigilância entomológica constante. Os estudos da fauna flebotomínica e de comportamento de algumas espécies são de grande relevância epidemiológica, pois podem fornecer dados que irão subsidiar a escolha, pelos órgãos competentes, dos métodos de controle da doença mais adequados à situação atual. Além disso, a verificação da taxa de infecção por Leishmania das espécies de flebotomíneos com potencial vetorial, nos focos periurbanos de Manaus merece atenção em investigações futuras.

Em relação à transmissão florestal deve-se aplicar medidas que reduzam o contato homem-vetor. Como uma maior densidade de vetores ocorre no período chuvoso, a adoção de medidas de proteção individual, como o uso de repelentes naturais de baixo custo e o uso de roupas que não exponham o corpo podem eventualmente ser úteis, embora esta última medida possa ser pouco utilizada no calor da Amazônia. A redução das atividades que envolvam manejo florestal neste período também é pouco factível para esse tipo de comunidade, pois o extrativismo vegetal faz parte de suas estratégias de subsistência. Na transmissão periflorestal o ideal seria construir as habitações com no mínimo 200m de distância da orla de floresta, além da telagem das casas e uso de mosquiteiros com redes de trama fina.

A aplicação de medidas de prevenção alternativas, baratas, práticas e que possam ser incorporadas no dia-a-dia das populações que vivem em áreas de risco devem ser propostas e avaliadas, pois estas estratégias podem ser aplicadas de maneira distinta para cada situação ou foco. Pressupõe-se que a prevenção e o controle das leishmanioses requerem estudos de avaliação da efetividade com componentes da promoção à saúde e participação imprescindível da comunidade em sua implementação, para assegurar sua sustentabilidade.

\section{AGRADECIMENTOS}

Aos técnicos de entomologia Flávio Augusto Andrade Fé, Orleans Furtado Pastor e ao motorista Elcimar Neves Cavalcante, pelo auxílio nas coletas de campo. Aos moradores da Comunidade São João, particularmente ao Mauro Silva, pelo auxílio durante a execução do Projeto.

\section{REFERÊNCIAS}

1. Aguiar GM, Medeiros WM. Distribuição e hábitats. In: Rangel EF, Lainson R (eds) Flebotomíneos do Brasil. Editora Fundação Oswaldo Cruz/Fiocruz , Rio de Janeiro, p. 207-255, 2003.

2. Andrade, SL, Fé NF, Fé FF, Toledo LM, Romero GAS. Leishmaniose tegumentar americana em área de ocupação recente na periferia da cidade de Manaus, estado do Amazonas, Brasil - Avaliação entomológica. Revista da Sociedade Brasileira de Medicina Tropical 32 (supl I): 11-12, 1999.

3. Arias JR, Freitas RA. Flebótomos da Amazônia Central do Brasil. I. Resultados obtidos das capturas feitas com iscas humana e eqüina (Diptera, Psychodidae). Acta Amazônica 7: 507-527, 1977.

4. Arias JR, Freitas RA. On the vectors of cutaneus Leishmaniasis in Central Amazon of Brazil. I. Preliminary findings. Acta Amazônica 12: 599-608, 1982.

5. Arias JR, Freitas RA. On the vectors of cutaneous leishmaniasis in Central Amazon of Brasil. III. Phlebotomine sandfly stratification in a terra firme forest. Acta Amazônica 12: 599-603, 1982.

6. Arias JR, Freitas RA. Sobre os vetores de leishmaniose cutânea na Amazônia central do Brasil. 2: Incidência de flagelados em flebótomos selváticos. Acta Amazônica 8: 387-396, 1978.

7. Arias JR, Freitas RA, Naiff RD, Barrett TV. Observations on the parasite Leishmania mexicana amazonensis and its natural infection of the sand fly Lutzomyia olmeca nociva. Bulletin of the Pan American Health Organization 21: 48-54, 1987.

8. Barrett TV, Freitas RA, Albuquerque MIC, Guerrero JHC. Report on a colection of Lutzomyia sand fly (Diptera: Psychodidae) from the Middle Solimões (Amazonas, Brasil). Memórias do Instituto Oswaldo Cruz 9: 27-35, 1996.

9. Barrett TV, Freitas RA, Naiff MF, Naiff RD. A leishmaniose e seus transmissores em relação à saúde na Amazônia. In: Val AL, Figlioulo R, Feldberg E (eds) Bases Científicas para Estratégias de Preservação e Desenvolvimento da Amazônia; Fatos e perspectivas. Instituto Nacional de Pesquisas da Amazônia /INPA, Manaus, p.150-117, 1991.

10. Barrett TV, Senra MS. Leishmaniasis in Manaus, Brazil. Parasitology Today 5: 255-257, 1989.

11. Cabanillas MRS, Castellón EGB, Alencar M. Estudo sobre os abrigos naturais dos flebotomíneos (Díptera: Psychodidae) na reserva florestal Ducke Manaus, AM, Brasil. Boletin de la Dirección de Malariología y Saneamiento Ambiental 35 (supl 1): 63-76, 1995.

12. Castellón EG, Fé NF, Buhrnheim PF, Fé FF. Flebotomíneos (Diptera, Psychodidae) na Amazônia. II. Listagem das espécies coletadas na Bacia Petrolífera no Rio Urucu, Amazonas, Brasil, utilizando diferentes armadilhas e iscas. Revista Brasileira de Zoologia 17: 455-462, 2000.

13. Desjeux $P$. The increase in risk factors for leishmaniasis worldwide. Transactions of the Royal Society of Tropical Medicine and Hygiene 95: 239-243, 2001.

14. Dias-Lima A, Castellon EG, Medeiros JF, Sherlock I. Estratificação vertical da fauna de flebótomos (Diptera, Psychodidae) numa floresta primária de terra firme da Amazônia Central, Estado do Amazonas, Brasil. Cadernos de Saúde Pública 8:823-832, 2002.

15. Feitosa MAC, Castellon EG. Fauna de flebotomíneos (Diptera: Psychodidae) em fragmentos florestais ao redor de conjuntos habitacionais na cidade de Manaus, Amazonas, Brasil. II. Estratificação horizontal. Acta Amazônica 34: 21-127, 2004.

16. Freitas RA, Barrett TV. Descriptions of Lutzomyia (Evandromyia) georgii $\mathrm{n}$. sp. and a Synopsis of the Series infraspinosa (Diptera: Psychodidae). Memórias do Instituto Oswaldo Cruz 97: 239-245, 2002.

17. Galati EAB. Morfologia e Taxonomia. In: Rangel EF, Lainson R (eds) Flebotomíneos do Brasil. Editora Fundação Oswaldo Cruz/Fiocruz , Rio de Janeiro, p. 23-51, 2003.

18. Gil LHS, Basano SA, Souza AA, Silva MGS, Barata I, Ishikawa EA, Camargo LMA, Shaw JJ. Recent observations on the sand fly (Diptera: Psychodidae) fauna of the State of Rondônia, Western Amazônia, Brazil: The importance of Psychodopygus davisi as a vector of zoonotic cutaneous leishmaniasis. Memórias do Instituto Oswaldo Cruz 98: 751-755, 2003.

19. Grimaldi Jr G, Momen H, Naiff RD, McMahon-Pratt D, Barrett TV. Caracterization and classification of leishmanial parasites from humans, wild mammals, and sand flies in the Amazon region of Brazil. The American Journal of Tropical Medicine and Hygiene 44: 645-661, 1991.

20. Grimaldi Jr G, Tesh RB, McMahon-Pratt D. A review of geographic distribution and epidemiology of leishmaniasis in the New World. The American Journal of Tropical Medicine and Hygiene 41: 687-725, 1989.

21. Guerra JAO, Barbosa MGV, Loureiro ACSP, Coelho CP, Rosa GG, Coelho LIAR. Leishmaniose Tegumentar Americana em crianças: aspectos epidemiológicos de casos. Cadernos de Saúde Pública 23: 2215-2223, 2007. 
22. Guerra JA0, Barros MLB, Guerra MVF, Talhari S, Paes MG. Leishmaniose Tegumentar Americana (LTA) no Município de Manaus - Aspectos epidemiológicos. Revista da Sociedade Brasileira de Medicina Tropical 31(supl I): 172, 1998.

23. Guerra JAO, Ribeiro JAS, Coelho LIAR, Barbosa MGV, Paes MG. Epidemiologia da leishmaniose tegumentar na Comunidade São João, Manaus, Amazonas, Brasil. Cadernos de Saúde Pública 22: 2319-2327, 2006.

24. Lainson R, Shaw JJ, Silveira FT, Souza AAA, Braga RR, Ishikawa EAI. The dermal Leishmaniasis of Brazil with special reference to the eco-epidemiology of the disease in the Amazonian. Memórias do Instituto Oswaldo Cruz 89: 435-443, 1994

25. Lainson R, Shaw JJ, Souza AAA, Silveira FT, Falqueto A. Further observations on Lutzomyia ubiquitalis (Psychodidae:Phlebotominae), the sandfly vector of Leishmania (Viannia) lainsoni. Memórias do Instituto Oswaldo Cruz 87: 437-439, 1992.

26. Lainson R, Ward RD, Shaw JJ. Cutaneous leishmaniasis in North Brazil: Lutzomyia anduze $i$ as a major vector. Transations of the Royal Society of Tropical Medicine and Hygiene 70: 171-172, 1976

27. Le Pont F, Desjeux P. Phlébotomes de Bolivie Description de Lutzomyia andersoni n. sp. (Diptera, Psychodidae). Memórias do Instituto 0swaldo Cruz 83: 421-425, 1988

28. Ministério da Saúde. Leishmaniose Tegumentar Americana - Distribuição de casos confirmados de LTA de 1980 a 2005. http://dtr2001.saude.gov.br/svsepi/ situacao_doencas/planilhas_doencas.htm (acessado em 12/03/2008).

29. Missawa NA, Giovana BMLM. List of species in the genus Lutzomyia, França, 1924 (Psychodidae, Phlebotominae) from the State of Mato Grosso. Revista da Sociedade Brasileira de Medicina Tropical 40: 11-14, 2007.

30. Paes MG. Estudo de quatro espécies de Lutzomyia França 1924 (Diptera Psychodidae) em área endêmica de Leishmaniose Tegumentar Americana na periferia de Manaus. Dissertação de Mestrado. Instituto Nacional de Pesquisas da Amazônia/Fundação Universidade do Amazonas, Manaus, AM, 1991.

31. Rangel EF, Lainson R Ecologia das Leishmanioses. In: Rangel EF, Lainson R (eds) Flebotomíneos do Brasil. Editora Fiocruz, Rio de Janeiro, p. 291-305, 2003.
32. Rangel EF, Ryan L, Lainson R, Shaw JJ. Observation on the sandfly (Diptera: Psychodidae) fauna of Além Paraíba, State of Minas Gerais, Brazil, and the isolation of a parasite of the Leishmania braziliensis complex from Psychodopygus hirsuta hirsuta. Memórias do Instituto Oswaldo Cruz 80: 373 374,1985 .

33. Ready PD, Lainson R, Shaw JJ. Leishmaniasis in Brazil: XX. Prevalence of "enzootic rodent leishmaniasis" (Leishmania mexicana amazonensis), and apparent absence of "pian bois" (Le. Braziliensis guyanensis), in plantations of introduced tree species and in other non-climax forests in eastern Amazônia. Transations of the Royal Society of Tropical Medicine and Hygiene 77: $775-785,1983$.

34. Ready PD, Lainson R, Shaw JJ, Ward RD. The ecology of Lutzomyia umbratilis Ward \& Fraiha (Diptera: Psychodidae), the major vector to man of Leishmania braziliensis guyanensis in north-eastern Amazonian Brazil. Bulletin of Entomological Research 76: 21-40, 1986.

35. Rebêlo JMM, Oliveira ST, Barros VLL, Costa JML, Ferreira LA, Silva AR. Phlebotominae (Diptera: Psychodidae) de Lagoas, município de Buriticupu, Amazônia Maranhense. I. Riqueza e abundância relativa das espécies em área de colonização recente. Revista da Sociedade Brasileira de Medicina Tropical 33: 11-19, 2000

36. Silva DF, Freitas RA, Franco AMR. Diversidade e abundância de flebotomíneos do gênero Lutzomyia (Diptera: Psychodidae) em áreas de mata do nordeste de Manacapuru, AM. Neotropical Entomology 36: 138-144, 2007.

37. Silveira FT, Souza AAA, Lainson R, Shaw JJ, Braga RR, Ishikawa EEA. Cutaneous Leishmaniasis in the Amazon Region: Natural infection of the sandfly Lutzomyia ubiquitalis (Psychodidae:Phlebotominae) by Leishmania (Viannia) lainsoni in Pará State, Brazil. Memórias do Instituto Oswaldo Cruz 86: 127-130, 1991.

38. Sudia WD, Chamberlain RW. Battery operated light trap, an improved model. Mosquito News 22: 126-129, 1962.

39. Thatcher VE. Studies of Phlebotomine Sandflies using castor oil traps baited with panamanian animals. Journal of Medical Entomology 5: 293-297, 1968 